\title{
Sample size calculation in economic RCTs: following clinical studies?
}

\author{
Sven Grüner ${ }^{(a)}$
}

March 09, 2019

\begin{abstract}
Clinical studies and economic experiments are often conducted utilizing randomized controlled trials. In contrast to clinical drug trials, sample size calculation has rarely been carried out by experimental economists. Using simple examples for illustration purposes, I discuss pros and cons of using sample size calculations in experimental economics.

\section{Keywords}

Economic experiments; sample size; multiple testing

JEL

C90; C10

(a) Martin Luther University Halle-Wittenberg, Institute of Agricultural and Nutritional Sciences, Chair of Agribusiness Management, Karl-Freiherr-von-Fritsch-Str. 4, 06120 Halle (Saale), Germany.
\end{abstract}




\title{
Sample size calculation in economic RCTs: following clinical studies?
}

\begin{abstract}
Clinical studies and economic experiments are often conducted utilizing randomized controlled trials. In contrast to clinical drug trials, sample size calculation has rarely been carried out by experimental economists. Using simple examples for illustration purposes, I discuss pros and cons of using sample size calculations in experimental economics.
\end{abstract}

\section{Keywords}

Economic experiments; sample size; multiple testing

JEL

C90; C10

\section{Introduction}

Randomization and double-blinding are the key design techniques in clinical trials. Blinding is used to mask which measure the patients receive. In practice, double-blinded studies are commonly utilized, where information that could reveal to which group the patients belong to is also withheld from the investigator. Randomization means random assignment of subjects to a treatment or the control group. The goal is to create groups with similar subjects, i.e. statistical independence between known and unknown confounders (e.g. age, gender, or other personal characteristics) and groups (e.g. Dunning 2012). Randomization achieves balance of covariates in expectation. However, in specific and especially small samples, randomization may be prone to not achieving a satisfactory covariate balance and thus jeopardizing valid causal inference.

A standard procedure in clinical drug trials is to calculate the optimal sample size before the study is carried out (e.g. Whitley and Ball 2002; Noordzij et al. 2010). If too few subjects are recruited (i.e. the study is underpowered) it may not be possible to detect a meaningful treatment difference between groups if such a difference exists (Nguyen and Landais 2017). Even small effects can be meaningful. The danger is that erroneous results will not give a reliable answer to the research question of the clinical trial. Whereas recruiting too many subjects (i.e. the study 
overpowered) leads to waste of resources, including monetary costs for both the experimenter and the sponsor of the study, and a possible delay in data collection. Ethical considerations may also be problematic. In contrast, the question of the optimal number of subjects has received much less attention in the social sciences. What can the methodical differences between clinical studies and experimental economics be attributed to? Randomized controlled clinical trials are rather expensive and directly linked to health of the patients (experimental subjects). In addition, clinical trials are aimed to make inference from the sample to the population. Thus, the negative consequences of erroneous results due to low external validity are far-reaching. In contrast, economists often carry out laboratory experiments with university students (Guala 2005; Levitt and List 2007). These experiments go hand in hand with low monetary costs and are easy to replicate. Looking for associations within the sample with no claim on generalizability is widely distributed among laboratory experiments (e.g. testing neoclassical assumptions and predictions). However, List et al. (2011) and Maniadis et al. (2014) are two important exceptions in which experimental economists use sample size calculations. Interestingly, one of the authors, John List, is a famous proponent of field experiments. Field experiments are much more similar to clinical studies (than laboratory experiments) because they are often cost intensive, not easy to replicate, and ethical considerations are an issue.

This paper explores the suitability of sample size calculations in the field of economic experiments. After providing some empirical evidence on the level of papers that use power calculations, I describe the necessary components and illustrate them with the help of two simple examples. Subsequently, pros and cons of using sample size calculation within experimental economics are discussed.

\section{Some empirical evidence on sample size analysis in economic experiments}

In this section I would like to illustrate the current practice of experimental studies in dealing with the calculation of the optimal number of subjects by means of the journal Experimental Economics. Experimental Economics is the leading field journal in the realm of economic experiments and, thus, reflects the state of the art of the discipline. I screened the issues from the very beginning of the journal in 1998 to 2018 for the keywords "power," "sample size," "calculation," and "subjects." It can be summarized that the bulk of studies neglect (ex ante) sample size calculations: the studies that actually carry out sample size calculations can be handpicked. In contrast to many experiments in psychology, subjects of controlled economic 
experiments usually receive monetary incentives. This may affect the number of participants. Ziegelmeyer et al. (2012) note that more participants would have increased the confidence in the robustness of their experiments, but were prevented due to financial budget limits. With some authors path dependency can be identified. For example, Candelo et al. (2018) state to have oriented themselves towards previous work in their research field when choosing the sample size.

Two early studies published in Experimental Economics address the term power in the context of statistical testing (Moir 1998; Feltovich 2003). Zelmer (2003) notes that the results of metaanalyses can be helpful for power calculations. In the period from 2007 to 2010 there are no power analyses in the published papers. A total of four studies conduct power analyses in the years 2011-2014 (i.e. a fraction of 3\% of the studies). List et al. (2011) provide valuable theoretical considerations on optimal sample arrangements. Stoop (2014) has performed power analyses since the "main result is based on the non-rejection of the null hypothesis." Somehow misleadingly the author seems to have carried out an ex-post calculation. In footnotes, Dreber et al. (2011) and Dreber et al. (2014) mention the required number of subjects at a significance level of $5 \%$ and a power of $80 \%$. In the period of $2015-2018$ there are five studies which conduct power analyses (slightly more than $3 \%$ of the studies). Jordan et al. (2016) briefly mention in a footnote that they carried out a power analysis. Koppel et al. (2017) use a previous study of their lab to determine the number of subjects to be recruited. However, the explanations are rather brief, for example there is no explanation why $70 \%$ power was chosen in their calculations. Utilizing prior knowledge through pilot experiments, Andersson et al. (2017) provide details on sample size calculation in their main study. Less straightforward appear to be two studies from 2018: Jacquemet et al. (2018) perform power analyses, however, the authors do not use prior knowledge but the data of their study. Filiz-Ozbay et al. (2018) were pointed to power calculations in the review process of their paper: "A referee raised the question of getting some idea of the increases in sample size that would be needed to determine if some of the Big 5 characteristics that had large, but statistically insignificant values, were in fact significant."1

\footnotetext{
${ }^{1}$ The quotation also illustrates what is currently strongly criticized in the literature: the dichotomy of "statistically non-significant"/"statistically significant." (e.g. Amrhein et al. 2019; [forthcoming] special issue of The American Statistician 73 about "a world beyond $\mathrm{p}<0.05$ ").
} 


\section{Sample size calculation-Parameters and experimental applications}

\subsection{Fundamental parameters of power analyses}

To conduct power analyses researchers must consider the following five parameters: Type I error, Type II error, MRD, and the variability of the outcome variable (e.g. Flurkey et al. 2007; Noordzij et al 2010; cf., Table 1):

- Potential for Type I error exists if a statistical test indicates differences between two groups. Type I error is the probability to falsely reject $\mathrm{H} 0$ (false positive result). It is usually denoted by the Greek letter $\alpha$ and often set at 0.05 .

- There is potential for Type II error if a statistical test does not indicate real differences between two groups. Type II error is the probability of falsely accepting H0 (false negative result), i.e. missing the real effect. It is usually denoted by the Greek letter $\beta$ and often set at 0.20. If negative findings ("no difference") are especially important for the conclusion of a study, lower $\beta$ 's are used (e.g. 0.1).

- Power is the (pre-study) probability of correctly rejecting H0 (true positive result), i.e. the chance of detecting the effect. It is inverse to Type II error (i.e. $1-\beta$ ). Power is often set at 0.8 (i.e. $\beta=0.2$ ) for a pivotal clinical trial. Power calculations have to be carried out before the data are collected. Ex-post calculations should not be performed because power is not about analyzing data (Senn 2002).

- Another variable to calculate the required sample size deals with the question of what difference the investigator wants to detect. The investigator needs information about the minimum difference regarded as important. Depending on the context, the minimal relevant difference (MRD) can either be clinically or economically. In other words, it is about the difference between treatment and control group (effect size of the treatment). This must be "clinically relevant and biologically plausible" (Noordzij et al. 2010:1389) in the context of medical studies, and economically relevant and plausible with spite of human behavior in the context of economic experiments. For this purpose, previous studies, former publications or expert opinions can be used. MRD is a numerical difference if the outcome variable is continuous, while event rates are used in case of binary outcomes.

- Moreover, assumptions about the variability of the sample data are required. These are initially unknown and must be taken from pilot studies or similar previous studies. Similarity 
relates to methods, subjects, and the design of the experiment. The variability of the sample data is measured in terms of population standard deviation if the outcome measure is continuous, or as a formula of event rates (depending on group assignment and outcome) if the outcome measure is binary.

Table 1 Parameters to calculate sample size

\begin{tabular}{|l|l|}
\hline$\alpha$ (Probability Type I error) & Probability, falsely rejecting H0 (=false positive result) \\
\hline$\beta$ (Probability Type II error) & Probability, falsely accepting H0 (=false negative result) \\
\hline Power $=1-\beta$ & (Pre-study) probability, correctly rejecting H0 (=true positive result) \\
\hline $\begin{array}{l}\text { Minimal } \\
{[\text { Clinically/Economically] }} \\
\text { Relevant Difference (=MRD) }\end{array}$ & $\begin{array}{l}\text { Difference between treatment and control group the investigator } \\
\text { wants to detect (=effect size of the treatment) }\end{array}$ \\
\hline Variability & Variability of the outcome variable \\
\hline
\end{tabular}

Determining parameters such as $\alpha$ and $\beta$ directly influences the result of the sample size calculation. Some researchers have recently proposed to use 0.005 instead of 0.05 as the default p-value threshold (cf., Benjamin et al. 2017). However, fixed thresholds are arbitrary to a certain extent in any case. Moreover, assumptions about the minimal relevant difference and the variability seem to be challenging. As it will be shown by simple examples in the following section and can be illustrated with the help of sensitivity analyses in which assumptions are systematically varied, even small changes can alter the required sample size substantially. However, thinking about the optimal sample size gives the investigator at least an idea under which circumstances more or less subjects are necessary to answer the research question.

\subsection{Calculating sample sizes in economics-Two simple (fictitious) experimental examples}

There are different kind of formulas to calculate sample size depending on the type of response variable (e.g. continuous, ordinal or binary), study design (e.g. cross-over trial, parallel group trial), and aim of the trial (e.g. superiority trials, non-superiority trials, non-inferiority trials) (Noordzij et al 2010; Christensen 2007). Performing power analyses is relatively straightforward and can be assisted by software packages (e.g. PASS, nQuery, G*Power, Sample Power).

I do not attempt to provide a comprehensive overview of these formulas because there exist many good overviews elsewhere. The interested reader may refer Julious (2004), Julious and Campbell 
(2012) or other standard literature in biomedical research. Instead, I would like to present the required components, briefly provide the intuition behind the calculations, and discuss whether this is a practical way in experimental economics. With this in mind, I illustrate sample size calculation with the help a randomized controlled trial design, in which are only two groups with equal sample size. ${ }^{2}$

\subsubsection{Continuous outcome variable}

If the outcome measure is continuous, the required sample size for each group reads as follows:

$$
N_{1,2}=\frac{2(a+b)^{2} \sigma^{2}}{\left(\mu_{1}-\mu_{2}\right)^{2}}
$$

To calculate the sample size one needs the standardized Z-scores (multipliers) for $\alpha$ (denoted as a in the formula above) and $\beta$ (denoted as $\mathrm{b}$ in the formula above). The multiplier for a can be both one-sided and two-sided. In two-tailed tests, the direction of the difference between the mean of the treatment group and the control group is not specified a priori. By contrast, with one-tailed test the direction is specified a priori. For example, the multiplier of $\alpha=0.05$ equals 1.96 (if the test is two-tailed) and 1.645 (if the test is one-tailed); the multiplier for $\beta=0.2$ equals 0.842 (cf., Table 2). For a given power, a larger sample size is required for two-tailed tests (Flurkey et al. 2007). If there is multiple testing, the probability of Type I error should be adjusted (Flurkey 2007). ${ }^{3}$ For example, the Bonferroni-adjustment proposes to divide 0.05 through the number of tests (cf., e.g. Holm 1979). After adjusting for multiple testing the standardized Z-score is obtained from the normal distribution. The difference of $\left(\mu_{1}-\mu_{2}\right)$ is the treatment effect the investigator wants to detect. Since this term is squared even small differences influence the sample size considerably. The same holds for the variance of the population $\sigma^{2}$. For continuous dependent variables the population standard deviation is used.

\footnotetext{
${ }^{2}$ Complexity increase with other designs. The formulas used in this section to calculate sample sizes are widely distributed among papers that deal with clinical trials or biomedical analyses. The can be found, for example, in Noordzij et al (2010: 1390) or Flurkey et al. (2007).

${ }^{3}$ Experimental economists often test multiple null hypotheses simultaneously (multiple treatments, subgroups, and outcomes). If researchers do not take account for them the evidence against the null is inflated. According to List et al. (2016) this problem is largely ignored among experimental economics studies.
} 
Table 2 Some multipliers for one- and two tailed tests

\begin{tabular}{|c|c|c|c|c|c|c|}
\hline \multirow{2}{*}{$\boldsymbol{\alpha}(\boldsymbol{a})$} & \multicolumn{3}{|c|}{ Two-tailed tests } & \multicolumn{3}{c|}{ One-tailed tests } \\
\cline { 2 - 7 }$\beta(\boldsymbol{b})$ & $\mathbf{0 . 1 0}$ & $\mathbf{0 . 0 5}$ & $\mathbf{0 . 0 1}$ & $\mathbf{0 . 1 0}$ & $\mathbf{0 . 0 5}$ & $\mathbf{0 . 0 1}$ \\
\hline $\mathbf{0 . 2}$ & $\mathbf{( 1 . 6 4 5 )}$ & $\mathbf{( 1 . 9 6 )}$ & $\mathbf{( 2 . 5 7 6 )}$ & $\mathbf{( 1 . 2 8 3 )}$ & $\mathbf{( 1 . 6 4 5 )}$ & $(\mathbf{2 . 3 2 6})$ \\
$(\mathbf{0 . 8 4 2})$ & 6.2 & 7.9 & 11.7 & 4.5 & 6.2 & 10.0 \\
\hline $\mathbf{0 . 1}$ & 8.6 & 10.5 & 14.9 & 6.6 & 8.6 & 13.0 \\
$\mathbf{( 1 . 2 8 3 )}$ & & & & & & \\
\hline
\end{tabular}

Note: The table depicts the multipliers (Z-scores) for conventional values of $\alpha$ (one-sided or two-sided) and $\beta$. For example, $\alpha=0.05$ (two-tailed test) equals 1.96. The multipliers of $\alpha$ and $\beta$ are denoted as $a$ and $b$, respectively. The calculations of $(\alpha+\beta)^{2}$ are provided in this table because they are necessary to calculate sample size. For more details see, for example, Snedecor and Cochran (1980:104) or Flurkey et al. (2007:659).

\section{Example: Dictator Experiment}

Let us assume that the experimenter would give $€ 100$ to player $A$. Player $A$ is then asked about the number of units he is willing to transfer to Player B. There are two treatments: (1) Player A and B are anonymous and cannot see each other, and (2) Player A and B can see each other. The investigator thinks that there is an inverse relationship between pro-social behavior and anonymity. Thus, he considers the amount of sharing to be higher in treatment 2 . The investigator wants to detect a difference of $€ 10$, which he supposes to be meaningful. Previous studies with similar subjects have shown that the variability is approximately normally distributed with a standard deviation of 25. Moreover, $\alpha$ and $\beta$ are assumed to be 0.05 and 0.2 , respectively.

It is assumed that the sum of money transferred to player B is higher in treatment 2. Thus, we use a one-tailed test. As it can be seen in Table 2, using the multipliers of $\alpha=0.05$ and $\beta=0.2$ gives $(\alpha+\beta)^{2}=(1.645+0.842)^{2}=6.2$. The variance $\sigma^{2}$ is 625 and the minimal relevant difference $\left(\mu_{1}-\mu_{2}\right)$ equals 10 . Putting these values into the formula above gives $N_{1,2}=$ $\frac{2 * 6.2 * 25^{2}}{(10)^{2}}=77.5$. To answer the research question, the required sample size for each group is 78 .

Doubling the minimal relevant difference from 10 to 20 reduces c.p. the required sample size to 
$N_{1,2}=19.375=20$. The investigator needs only $1 / 4$ of the subjects (differences are due to rounding ${ }^{4}$ ). Halving the standard deviation from 25 to 12.5 leads to the same conclusion.

\subsubsection{Binary outcome variable}

If the outcome measure is binary, the required sample size for each group reads as follows:

$$
N_{1,2}=\frac{(a+b)^{2}\left(f_{1} * \overline{f_{1}}+f_{2} * \bar{f}_{2}\right)}{\mu^{2}}
$$

Similar the continuous outcome scenario described earlier, the standardized Z-scores for $\alpha(=a$ in the formula above) and $\beta$ (=b in the formula above) have to be considered to calculate the sample size. Two groups and outcomes have to be distinguished: $f_{1}\left(\bar{f}_{1}\right)$ denotes the fraction of people with (without) outcome 1 in treatment group 1 , whereas $f_{2}\left(\bar{f}_{2}\right)$ denotes the fraction of people with (without) outcome 1 in treatment group 2. The difference the investigator supposes to be meaningful and wants to detect is captured by $\mu$. This term is squared and has an important influence on the sample size calculation. For binary dependent variables the event rates are used.

\section{Example: One-shot prisoner's dilemma}

The investigator conducts a one-shot prisoner's dilemma game. He wants to examine whether information about the opponent influences the decision to cooperate or defect. In treatment 1 there is no information about the opponent, whereas in treatment 2 it is known that the opponent raised in rural areas. Suppose that the investigator knows from earlier studies that $30 \%$ of the subjects cooperate without having any information about their opponent, whereas $40 \%$ of the subjects tend to cooperate if the opponent raised in rural areas. The investigator deems a difference of $20 \%$ in cooperation propensity to be meaningful. Moreover, $\alpha$ and $\beta$ are assumed to be 0.05 and 0.2 , respectively. The investigator wants to run a two-sided test.

Assuming a two-sided test gives $(a+b)^{2}=(1.96+0.842)^{2}=7.9$. The fraction of subjects who cooperate is captured by $f_{1}$ and $f_{2}$ and non-cooperation is labeled as $\bar{f}_{1}$ and $\bar{f}_{2}$, with the lower superscript numbers indicating the number of the treatment group. $f_{1} * \bar{f}_{1}+f_{2} * \bar{f}_{2}=$ $0.4(1-0.4)+0.3(1-0.3)=0.45$. The minimal relevant difference $\mu^{2}$ is $0.2^{2}=0.04$. The formula to calculate the required sample size for each group gives $N_{1,2}=\frac{7.9 * 0.45}{0.04}=88.875$. To answer the research question 89 subjects should be recruited for each of the groups. If we would halve the difference deemed to be meaningful to 0.1 , the required sample size would be

\footnotetext{
${ }^{4}$ It is important to be cautious with the sample size and in any doubt to recruit the higher number of subjects. For example, in practice the investigator should round up the number of subjects to answer the research question.
} 
quadrupled. A total of 356 subjects per group (the unrounded number is 355.5) would be required to answer the research question.

\section{Discussion}

Do we need sample size calculations in lab experiments or is it sufficient to aggregate evidence through replication? Filiz-Ozbay et al. (2018) argue that researchers put much more effort in sample size calculations in field experiments because lab experiments could be replicated easily. In some cases extensive replications can be observed. For example, the Ultimatum bargaining game, introduced by Güth et al. (1982), has been replicated many times. Another example is the experimental work of Kahneman and Tversky. There are many robustness checks of their initial studies, which makes it easy to conduct systematic reviews and meta analyses - a starting point for further research. What experiments are likely to be replicated? Probably the ones that are easy to replicate and do not cause much costs. Overall the incentives to replicate experiments are relatively low (Galiani et al. 2017). Camerer et al. (2016) provide evidence for the insufficient reproducibility of studies in the empirical social sciences. They attempted to replicate 18 economic experiments, published in the American Economic Review and The Quarterly Journal of Economics in the 2011-2014 journals. The authors find statistically significant effects with the same signs for only 11 of the replicated studies. On average the effect sizes in the replication studies was significantly lower than in the original studies. Furthermore, there are studies that have been replicated infrequently (e.g. field experiments are often rather expensive and hard to replicate). Such studies can provide important impulses. But the researcher must take care not to overinterpret their findings, especially when the sample size is low or not even calculated before the data was collected. This is not an easy task-even for Nobel laureates. For example, Kahneman admitted that he "[...] placed too much faith in underpowered studies." 5 Therefore, replication cannot fully serve as substitute for sample size calculation.

If there is no solid data basis (e.g. in case of an entirely new experiment) sample size calculations cannot be done properly until pilot studies are carried out or reliable expert estimates are available. Of course, studies without clear pre-specified hypotheses should be identified as such. For example, Berry (2016) proposes to explicitly declare them as exploratory and to point out that there is no claim of generalizability. Cooper et al. (1999) distinguish between hypotheses that were specified before the data was collected (ex ante hypotheses) and hypotheses that were

\footnotetext{
${ }^{5}$ See, for example: https://retractionwatch.com/2017/02/20/placed-much-faith-underpowered-studies-nobel-prizewinner-admits-mistakes/
} 
discovered after seeing the data (ex post hypotheses). The former can be classified as confirmatory und the latter exploratory analysis.

There is at least one indirect, but crucial advantage attached to sample size calculations. Thinking about the required sample size necessitates to make precise assumptions about both the null and the alternative hypothesis. While the former should "capture the status quo line of thinking" (Brandon and List 2015), it is often unrealistically assumed to be zero in economics regression analysis. But this disregards prior knowledge and former research findings. ${ }^{6}$ Besides methodical similarities (e.g. using RCTs) sample size calculations are standard practice in clinical, but not economic trials. As described above there are some good arguments to conduct them in experimental economics research, too. One of the main arguments is objectivity of research. If other scientists read a paper, it is important for them to be informed about issues like power and the optimal sample size. Published research is at least input for new studies and we need to know how to evaluate them.

\section{Acknowledgment}

Funded by the Deutsche Forschungsgemeinschaft (DFG, German Research Foundation) 388911356.

\section{References}

Andersson, O., Miettinen, T., Hytönen, K., Johannesson, M., Stephan, U. (2017): Subliminal influence on generosity. Experimental Economics 20(3): 531-555.

Amrhein, V., Greenland, S., McShane, B.B. (2019): Retire statistical significance. Nature (forthcoming).

Bacchetti, P. (2010): Current sample size conventions: Flaws, harms, and alternatives. BMC Medicine 8: 17.

Benjamin, D.J et al. (2017): Redefine Statistical Significance. Human Nature Behavior. https://www.nature.com/articles/s41562-017-0189-z

Berry, D.A. (2016): P-Values Are Not What They're Cracked Up to Be. Online Discussion: ASA Statement on Statistical Significance and P-values. The American Statistician 70(2): 1-2.

\footnotetext{
${ }^{6}$ To be fair, clinical investigators have a comparative advantage to economists. Economists do have databases to search for literature (e.g. Web of Science, RePeC), but they are not in that luxurious situation to have access to high level established standards. Clinical investigators can use PubMed to have an easy and efficient access to biomedical literature.
} 
Brandon, A., List, J.A. (2015): Markets for replication. Proceedings of the National Academy of Sciences of the United States of America 112(50): 15267-15268.

Camerer, C.et al. (2016): Evaluating replicability of laboratory experiments in economics. Science 351: 1433-1436.

Candelo, N., Croson, R.T.A., Eckel, C. (2018): Transmission of information within transnational social networks: a field experiment. Experimental Economics 21(4): 905-923.

Christensen, E. (2007): Methodology of superiority vs. equivalence trials and non-inferiority trials. Journal of Hepatology 46(5): 947-954.

Cooper, D.J., Kagel, J.H., Lo, W., and Gu, L.Q. (1999): Gaming Against Managers in Incentive Systems: Experiments with Chinese Students and Chinese Managers. American Economic Review 89(4): 781-804.

Dreber, A., von Essen, E., Ranehill, E. (2011): Outrunning the gender gap-boys and girls compete equally. Experimental Economics 14(4): 567-582.

Dreber, A., von Essen, E., Ranehill, E. (2014): Gender and competition in adolescence: task matters. Experimental Economics 17(1): 154-172.

Dunning, T. (2012): Natural Experiments in the Social Sciences. A Deisgn-Based Approach. Cambridge University Press: Cambridge.

Feltovich, N. (2003): Nonparametric Tests of Differences in Medians: Comparison of the Wilcoxon-Mann-Whitney and Robust Rank-Order Tests. Experimental Economics 6(3): 273-297.

Filiz-Ozbay, E., Ham, J.C., Kagel, J.H., Ozbay, E.Y. (2018): The role of cognitive ability and personality traits for men and women in gift exchange outcomes. Experimental Economics 21(3): 650-672.

Flurkey, K., Currer, J.M., Harrison, D.E. (2007): Mouse Models in Aging Research. In: Fox et al. The Mouse in Biomedical Research 3: 637-672.

Galiani, S., Gertler, P., Romero, M. (2017): Incentives for replication in economics. Tech. rept. National Bureau of Economic Research. https://www.nber.org/papers/w23576.pdf

Guala, F. (2005): The Methodology of Experimental Economics. Cambridge University Press, Cambridge.

Güth, W., Schmittberger, R., Schwarze, B. (1982): An experimental-analysis of ultimatum bargaining. Journal of Economic Behavior \& Organization 3(4): 367-388.

Holm, S. (1979): A simple sequentially rejective multiple test procedure. Scandinavian Journal of Statistics 6(2): 65-70.

Jacquemet, N., Luchini, S., Shogren, J.F., Zylbersztejn, A. (2018): Coordination with communication under oath. Experimental Economics 21(3): 627-649.

Jordan, J., McAuliffe, K., Rand, D. (2016): The effects of endowment size and strategy method on third party punishment. Experimental Economics 19(4): 741-763.

Julious, S.A. (2004): Tutorial in Biostatistics. Sample sizes for clinical trials with Normal data. Statistics in Medicine 23(12): 1921-1986. 
Julious, S.A., Campbell M.J. (2012): Tutorial in biostatistics: sample sizes for parallel group clinical trials with binary data. Statistics in Medicine 31(24): 2904-2936.

Koppel, L., Andersson, D., Morrison, I., Posadzy, K., Västfjäll, D., Tinghög, G. (2017): The effect of acute pain on risky and intertemporal choice. Experimental Economics 20(4): 878-893.

Levitt, S.D., List, J.A. (2007): What Do Laboratory Experiments Measuring Social Preferences Reveal about the Real World? The Journal of Economic Perspectives 21(2): 153-174.

List, J.A., Sadoff, S. \& Wagner, M. (2011): So you want to run an experiment, now what? Some simple rules of thumb for optimal experimental design. Experimental Economics 14: 439457.

List, J.A., Shaikh, A.M., Xu, Y. (2016): Multiple Hypothesis Testing in Experimental Economics. NBER Working Paper No. 21875.

Maniadis, Z., Tufano, F., List, J. (2014): One Swallow Doesn't Make a Summer: New Evidence on Anchoring Effects. American Economic Review 104(1): 277-290.

Moir, R. (1998): A Monte Carlo Analysis of the Fisher Randomization Technique: Reviving Randomization for Experimental Economists. Experimental Economics 1(1): 87-100.

Noordzij, M., Tripepi, G., Dekker, F.W., Zoccali, C., Tanck, M.W., Jager, K.J. (2010): Sample size calculations: Basic principles and common pitfalls. Nephrol Dial Transplant 25: $1388-1393$.

Nguyen, T.-L., Landais, P. (2017): Randomized controlled trials: significant results-fragile, though. Kidney International 92(6): 1319-1320.

Senn, S.J. (2002): Power is indeed irrelevant in interpreting completed studies. British Medical Journal 325(7375): 1304.

Snedecor, G.W., Cochran, G.W. (1980): Statistical methods. The Iowa State University Press: Ames.

Stoop, J. (2014): From the lab to the field: envelopes, dictators and manners. Experimental Economics 17(2): 304-313.

Zelmer, J. (2003): Linear Public Goods Experiments: A Meta-Analysis. Experimental Economics 6(3): 299-310.

Ziegelmeyer, A., Schmelz, K., Ploner, M. (2012): Hidden costs of control: four repetitions and an extension. Experimental Economics 15(2): 323-340. 